\title{
Flight Testing of an Structured H-infinity Controller: An EU-Japan Collaborative Experience
}

\author{
Andrés Marcos ${ }^{1}$ and Masayuki Sato ${ }^{2}$
}

\begin{abstract}
This article presents the results from the first flight campaign undertaken within the frame of a collaborative EU-Japan project aimed towards the technological maturation of advanced aircraft guidance, navigation and control techniques. A control design (developed and flight tested by JAXA several years ago) was selected as the baseline system and objectives. This baseline design was based on a two degrees-offreedom structure aimed at gust suppression in the feedback part and handling qualities model-matching objectives in the feedforward part. A newly developed controller uses this legacy interconnection and objectives and applies the Structured Hinfinity control design approach with the goal to demonstrate the latter's potential to fulfill the same objectives while reducing the controller's dimension. The flight tests results presented show that this was successful and that both controllers' behavior are mostly indistinguishable, but with the new controller at half the dimension of the baseline.
\end{abstract}

\section{INTRODUCTION}

"Smart" systems integrating vision-based, fault detection/isolation (FDI) and fault tolerant control (FCT) technologies are expected to be key towards achieving a meaningful reduction in the current level of aircraft accidents. Many of these technologies have attained a sufficiently mature technological readiness level (TRL) but few concerted efforts have been carried out to mature their integrated operation, which is fundamental to transfer them to industry for operational deployment. Furthermore, due to the criticality of validating fault-related scenarios on aircraft, even less number of projects have been able to increase the TRL of the individual technologies to a level that the aeronautical industry can rely on. In Europe there has been a continuous push to evolve and mature the FDI/FTC domain for aircraft since 2005 with the GARTEUR projects' RECOVER [20] to the EU FP6 PEGASE [17] and then the two consecutive EU FP7 projects ADDSAFE [7] and RECONFIGURE [8]. The perspective taken in Europe was to align the academic and industrial research groups to increase the TRL in a "industrial" manner, i.e. in incremental steps following the aeronautical industry Verification \& Validation $(\mathrm{V} \& \mathrm{~V})$ cycle. With a similar goal but following a "testing" approach, i.e.

\footnotetext{
*This work was funded by the European Union's Horizon 2020 (H2020) programme under grant agreement No. 690 811, and Japan's New Energy and Development Organization (NEDO) under grant agreement No. 062800. It is a part of the EU/Japan joint research project entitled "Validation of Integrated Safety-enhanced Intelligent flight cONtrol (VISION)"

${ }^{1}$ Andrés Marcos is the leader of the Technology for Aerospace Control (TASC) lab, http://www.tasc-group.com/, at the Aerospace Engineering Department, University of Bristol, BS8 1TR, U.K. andres.marcosebristol.ac.uk

${ }^{2}$ Masayuki Sato is with the Institute of Space Technology and Aeronautics, Japan Aerospace Exploration Agency, Mitaka, Tokyo 181-0015, Japan sato.masayuki@jaxa.jp
}

maturing advanced technologies through fly testing (but with no emphasis on industrial considerations), Japan funded a series of projects starting in 2002 with AFCG [18] and concluding in 2010 with IFTC [19]. The core of this flight testing was carried out by JAXA due to their ownership of a Fly-By-Wire (FBW) research aircraft, MuPAL- $\alpha$ [1].

Recently, February 2016, a three-year project entitled "Validation of Integrated Safety-enhanced Intelligent flight cONtrol (VISION)", has been established with funding by the European Union Horizon 2020 program (EU-H2020) and Japan's New Energy and Development Organization (JPNEDO). The goal of this project is precisely to mature the aforementioned "smart" technologies by exploiting the complementariness of the efforts by EU (ample experience with industrial constraints and transfer of FDI/FTC) and Japan (strong flight testing experience of advanced control designs, as well as availability of MuPAL- $\alpha$ ).

This article presents the results from the first flight campaign of the H2020 EU-NEDO JAPAN VISION project. This first campaign focused on establishing the collaboration between the University of Bristol (UoB) and JAXA teams in as much as performing the flight testing of an advanced control approach. As such, a control design (developed and flight tested by JAXA several years ago) was selected as the baseline [14]. It is based on a two degrees-of-freedom structure aimed at gust suppression in the feedback part and handling qualities model-matching objectives in the feedforward part. The newly developed controller uses this legacy interconnection and objectives and applies the structured $H_{\infty}$ control design approach [13] with the goal to demonstrate the latter's potential to fulfill the same objectives but reducing the controller's dimension. The flight tests results presented show that this was successful and that both controllers' behavior are mostly indistinguishable, but with the new controller at half the dimension of the baseline.

The layout of the article is as follows. Section II presents JAXA's MuPAL- $\alpha$ and the baseline controller. Section III presents the new control design obtained using the structured $H_{\infty}$ control design approach. Section IV presents the V\&V of both controllers, from analysis to verification and then validation (i.e. flight tests). Finally, Section V presents the main conclusions.

\section{JAXA MUPAL- $\alpha$ AND BASELINE CONTROL DESIGN}

In this section, JAXA's research aircraft MuPAL- $\alpha$ is described first, and is followed by a cursory presentation of the baseline controller used in this work but developed and flight tested by JAXA in 2011. 


\section{A. JAXA's fixed-wing FBW research aircraft}

Japan Aerospace Exploration Agency (JAXA) has a fleet of research aircraft encompassing fixed-wing turbo-prop and jet aircraft, as well as a rotor craft. These aircraft have been used for example: to perform handling quality investigation [2], parameter identification [3], and Instrument Flight Rules (IFR) flight examination [4].

In particular, the fixed-wing turbo-prop aircraft known as Multi-Purpose Aviation Laboratory- $\alpha$ (MuPAL- $\alpha$ ) [1], shown in Fig. 1, has the ability to conduct various advanced control research studies thanks to the flexibility of its experimental FBW system (running at $50 \mathrm{~Hz}$ ). During flight tests, MuPAL- $\alpha$ is operated by two pilots, a safety pilot (SP) in charge of flying from/to the test area and take over during tests if any issue arises, and an evaluation pilot (EP) in charge of carrying out the tests. The aircraft is actuated by one set of ailerons, a single elevator, and a rudder but also by socalled Direct Lift Controls (DLC) flaps -which provide an additional longitudinal actuation capability. There are airdata sensors (ADS/ADC running at 12.5/25/50 Hz) and GPS/INS measurements (available at 5/50/200 Hz).

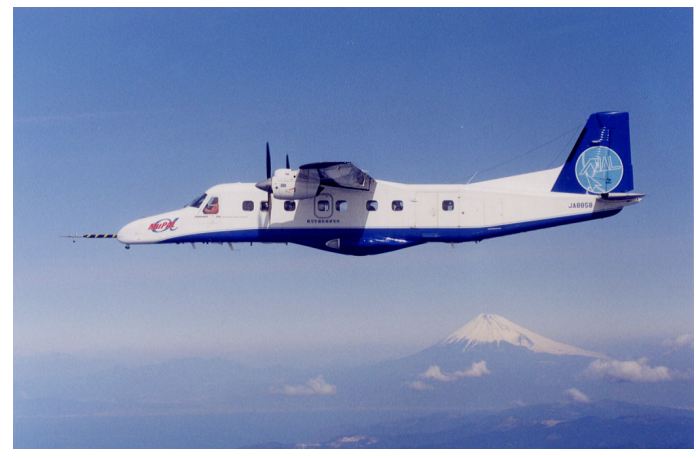

Fig. 1: JAXA's research aircraft MuPAL- $\alpha$

\section{B. Plant 2-DoF control design}

In reference [14], state-space plants of MuPAL- $\alpha$ for the longitudinal and lateral-directional (lat/dir) motions are given for a wings-level flight condition at a true-airspeed of $V_{T A S}=$ $69.8[\mathrm{~m} / \mathrm{s}](136 \mathrm{kts})$. These plants are used to design a 2 Degrees-Of-Freedom (DoF) control system for each motion, which was successfully flight tested. These lat/dir data and controller will be used as the baseline for comparison.

In this article, the focus is on the lat/dir motion, and from the previous reference the associated control design objectives are generally defined as:

- Gust suppression

- Handling qualities model-matching

The two objectives must be achieved robustly against actuator modeling errors represented by uncertain time delay ranges. Note that for the second objective, the capability of MuPAL- $\alpha$ to serve as an In-Flight Simulator (IFS) [5] is exploited. An IFS is an aircraft which can mimic another aircraft's characteristics (i.e. handling qualities, gust response, and even on-board equipment operation).

The lat/dir plant dynamics have four states: sway inertial speed $v_{i}[\mathrm{~m} / \mathrm{s}]$, roll rate $p[\mathrm{rad} / \mathrm{s}]$, roll angle $\phi[\mathrm{rad}]$ and yaw rate $r[\mathrm{rad} / \mathrm{s}]$; one disturbance input $v_{g}[\mathrm{~m} / \mathrm{s}]$ (sway wind gust) and two control inputs $\left[\delta_{a} \delta_{r}\right]^{T}[\mathrm{rad}]$ (aileron and rudder deflection respectively), and their commanded counterparts $\left[\begin{array}{ll}\delta_{a c} & \delta_{r c}\end{array}\right]^{T}$ ). The measurement output vector is given by $\left[\begin{array}{llll}v_{a} & p & \phi & r\end{array}\right]^{T}$ where $v_{a}[\mathrm{~m} / \mathrm{s}]$ represents sway airspeed.

The control inputs are driven by on-board actuators with dynamics modelled by first order systems in series with pure delays (but with an uncertain time value):

$$
\delta_{a}=e^{-T_{a} s} \frac{0.67}{0.12 s+1} \delta_{a_{c}} ; \quad \delta_{r}=e^{-T_{r} s} \frac{0.74}{0.07 s+1} \delta_{r_{c}}
$$

The uncertain time delays $T_{a}$ and $T_{r}$ can range from a minimum value of 0.06 to a maximum of 0.4 and 0.2 seconds respectively. For the controller design they are implemented as first-order Padé approximation models described as linear fractional transformations (see reference [14] for details).

As aforementioned, JAXA's (the baseline) controller is a 2-DoF control system, see Fig. 2, composed of: a feedback component $K$, a feedforward component $F$, and a model $M$ of the aircraft to be mimicked. The feedback component $K$ was designed first to achieve the robust disturbance suppression objective, while the feedforward component $F$ was designed afterwards to fulfil the model-matching objective (to the handling characteristic of an aircraft represented by $M$ ). The case of a B747 in steady-level flight at $V_{T A S}=67.4[\mathrm{~m} / \mathrm{s}]$, altitude of $0[\mathrm{~m}]$, and without a Stability Augmentation System (SAS) that was presented in [14] is also used in this article.

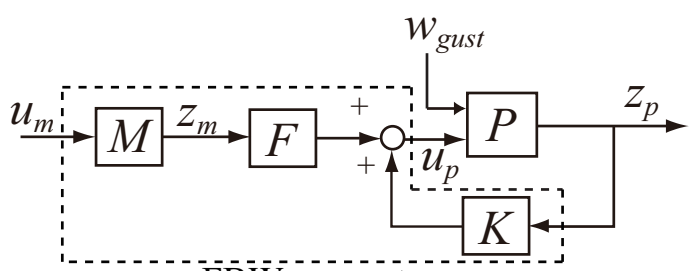

FB $\bar{W}$ computer

Fig. 2: JAXA-K: 2-DoF controller structure

The feedback controller $K$ was designed using a cone complementarity linearization algorithm [6] that allowed for low-order controllers in order to address on-board computers' numerical complexity. The final dimension was 1 as a static controller could not be found that fulfilled the design requirement - given with respect to Fig. 3 as:

$$
\sup _{\omega \in \mathbb{R}} \mu_{\operatorname{diag}\left(\Delta_{u}, \Delta_{p_{K}}\right)}\left(G_{z w}(j \omega)\right)<1
$$

In Fig. 3, $w_{u}$ and $z_{u}$ are signals representing the onboard actuators' uncertain delays, $z_{p}\left(=\left[\begin{array}{ll}\phi & r\end{array}\right]^{T}\right)$ denotes the performance output, $y_{p}$ denotes the measurement output and $u_{f b}$ the feedback controller output. In addition, $\Delta_{u}$ and $\Delta_{p_{K}}$ denote respectively the delay uncertainty and the fictitious performance blocks, the former arising from $P$, the plant model represented as a Linear Fractional Transformation (LFT) for the approximated delay models. The design weighting function $W_{1}$ was defined as a constant matrix to obtain a controller of low dimension:

$$
W_{1}=\operatorname{diag}(1.44,0.72) \text {. }
$$




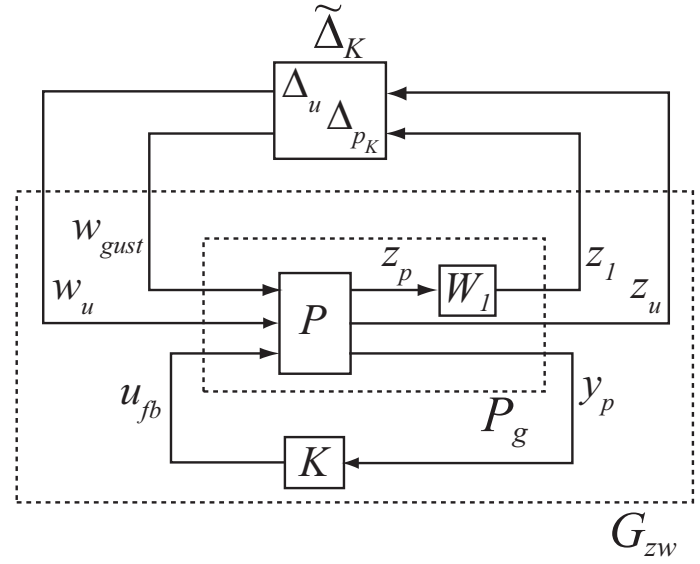

Fig. 3: JAXA-K: Feedback $K$ design interconnection

Similarly, a full-order stable feedforward controller $F$ of dimension 11 was then designed based on Fig. 4 (where $P_{n}$ denotes the plant model assuming nominal actuator delays, i.e. $T_{a}=0.23$ and $T_{r}=0.13$ ) to satisfy the requirement:

$$
\left\|G_{z_{2} w_{2}}(s)\right\|_{\infty}<1
$$

For this case, the weighting function $W_{2}$ was defined to realize the inverse property of $P_{c l}$ in the low frequency range:

$$
W_{2}=\mathbf{I}_{2} \otimes \frac{7.1}{s+0.35} .
$$

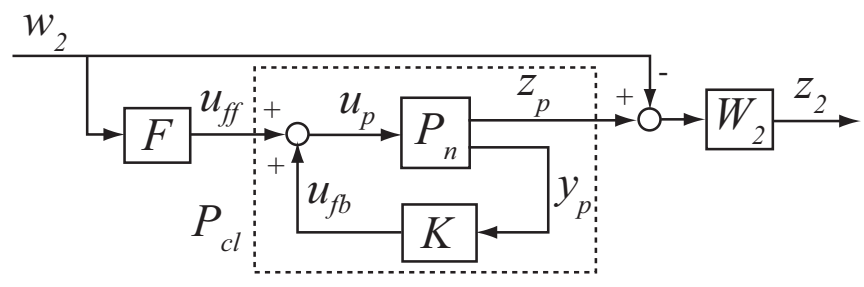

Fig. 4: JAXA-K: Feedforward $F$ design interconnection

\section{STRUCTURED H-INFINITY CONTROL DESIGN}

A structured $H_{\infty}$ controller design approach based on non-smooth optimization has been proposed in [13], and its effectiveness has been demonstrated already in several simulation design problems, e.g. [10], [11], [12].

The four main motivations for using a structured $H_{\infty}$ control design approach are:

i to keep controller's dimension low directly from design,

ii to use optimization to select the controller's parameters, iii to consider sets of models and/or design constraints, iv to specify the structure of the controller.

This method is used by the University of Bristol (UoB) to design a controller following the 2-DoF architecture, models and goals detailed in the previous section (i.e. those by JAXA for the baseline controller). The aim is the sequential design of the feedback and feedforward components towards achieving the same objectives as JAXA but using structured $H_{\infty}$ to reduce their complexity (i.e. dimension) and show the potential of this technique to use legacy information. Indeed, the idea for UoB's controller is to re-use the formulation and weighting functions from [14] as much as possible. It is noted that this is the first time that such a type of controller is flight tested (in Space, there are two instances where this technique has been used to tune and/or develop a controller [15], [16]).

A departure from [14] in the design process is that for the structured $H_{\infty}$ design of each component a set of three time delays are used simultaneously (set at the minimum, nominal and maximum values of their ranges).

\section{A. FeedBack design, $K_{F D}$}

For the design of the feedback controller, see Fig.5, the structured $H_{\infty}$ design interconnection uses two design weights: an output weight $W_{\text {lout }}=\operatorname{diag}(1.1,1.1,1,1,1,1)$ (normalized to radians, and initially based on that from JAXA) and an input weight $W_{1 \text { out }}=\operatorname{diag}(1,1,1.2)$. They are pre/post- appended to the augmented plant consisting of the lat/dir motion plant (with gust input) in series with the actuator and the uncertain time delay models. A diagonal block matrix is then formed condensing all the weighted augmented plants (recall that three different delay models are used) and finally the hinfstruct command is used to obtain the feedback controller.

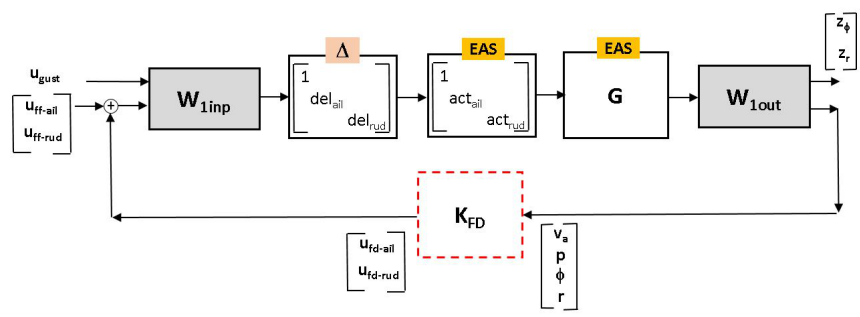

Fig. 5: UoB-K: feedback $K_{F D}$ design interconnection

The structured $H_{\infty}$ method gives after 5 iterations a static controller (i.e. a gain matrix with four inputs and two outputs) with a design $\gamma$ of 1.09. Notice that using the method of [14] it was not possible to obtain a static controller.

\section{B. FeedForward design, $K_{F F}$}

For the feedforward, see Fig. 6, a similar process is used but the augmented plant does not have gust inputs, includes the feedback controller, and only uses the following output weight on the performance error channels:

$$
W_{2}=\operatorname{diag}(0.65,1.2) \otimes \frac{7.1}{s+0.35} .
$$

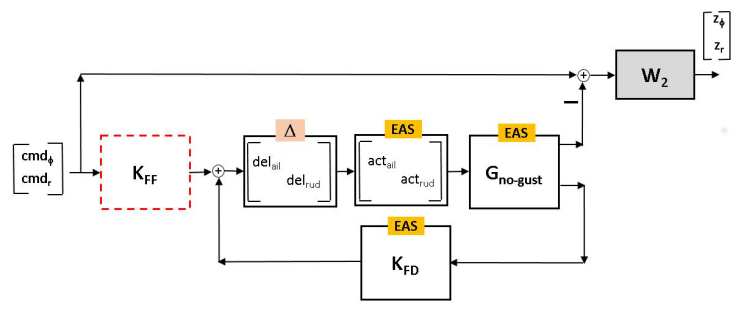

Fig. 6: UoB-K: Feedforward $K_{F F}$ design interconnection

After 6 iterations and a $\gamma$ of 2.12, a 6 state controller is obtained. As above, notice that the resulting UoB controller 
is half the number of states of JAXA's design on which it is based (but also note that the difficult design step of setting the design interconnection and initial weights was performed during JAXA's design). The value $\gamma>1$ means the objectives were not fully achieved, this can be due to an inadequate weight normalization or too challenging demands. As shown next it did not affect the good performance of the design.

\section{CONTROL VERIFICATION AND VALIDATION}

A standard Verification and Validation $(\mathrm{V} \& \mathrm{~V})$ campaign involves three main phases: (i) analysis, (ii) verification in Hardware-In-The-Loop (HILS), and (iii) validation in flight test. Each phase must be successfully performed before the next phase is attempted, and each uses an incremental sophistication in the tools, models and test-benches. A good overview of the industrial $\mathrm{V} \& \mathrm{~V}$ steps is given in [7].

\section{A. Design Analysis}

For the design analysis phase, the baseline and UoB controllers are analyzed using a standard set of linear frequency (FRSP) and time (TRSP) responses, and including polezero maps of the open-loop, inner-loop (plant and feedback component) and closed-loop (CLP, i.e. the full setting of Fig. 2 ). For the analysis of the baseline refer to [14].

Fig. 7 shows the FRSP from the aileron and rudder commands to the performance outputs $\left[\begin{array}{ll}\phi & r\end{array}\right]^{T}$, and including also the transfer functions from the gust input to the latter (in solid green in the main diagonal plots), for EAS speeds from 100 to 140 knots every 20 knots.
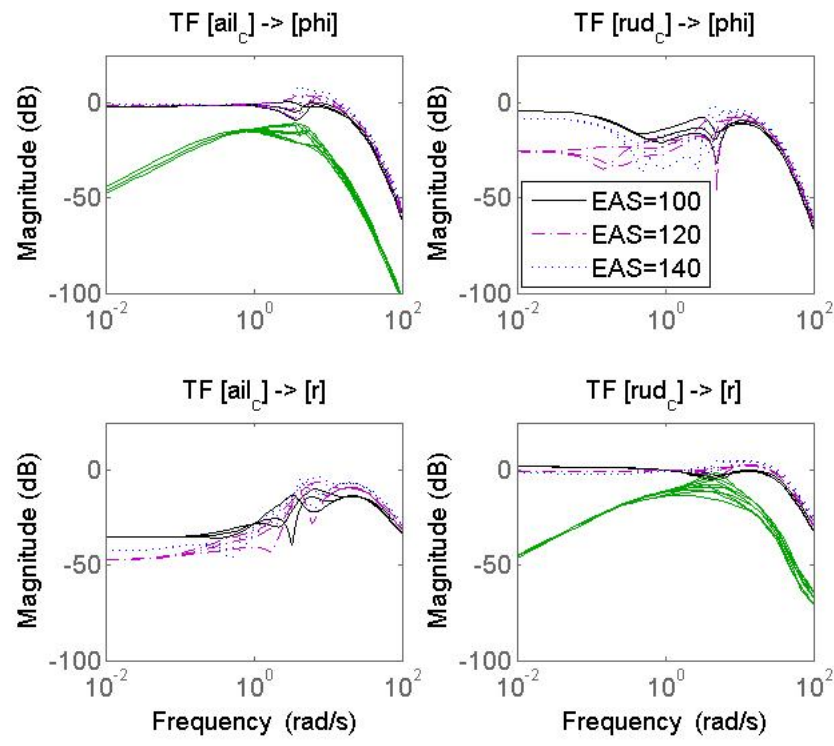

Fig. 7: FRSP UoB-K: closed-loop tracking and coupling for $E A S=[100: 20: 140] \mathrm{kts}$, no time delay uncertainty $\Delta$

It is seen that the tracking is adequate (the complimentary sensitivity plots in the main diagonal plots) and that the gust rejection is adequate (the green lines in the main diagonal). Similarly, it is easily seen that the coupling from aileron to yaw rate (bottom-left plot) is better than that for the rudder to roll angle, which shows DC gains close or above $0 \mathrm{~dB}$ for $\mathrm{EAS}=[110,140] \mathrm{kts}$.
Fig.8 shows the linear TRSP of the closed-loop against a square gust perturbation from 2 to 15 seconds (the black dashed line in the top plot) for five EAS (from 100 to 140 kts, every 20 knots). Note that for each speed, 15 perturbed plants are used (i.e. the uncertain delay models are fixed at 15 randomly selected values within their range). It is seen that the responses and commands are all very tight and adequate. The same TRSP for the baseline controller yielded also acceptable responses but noticeably more oscillatory for the performance channels.

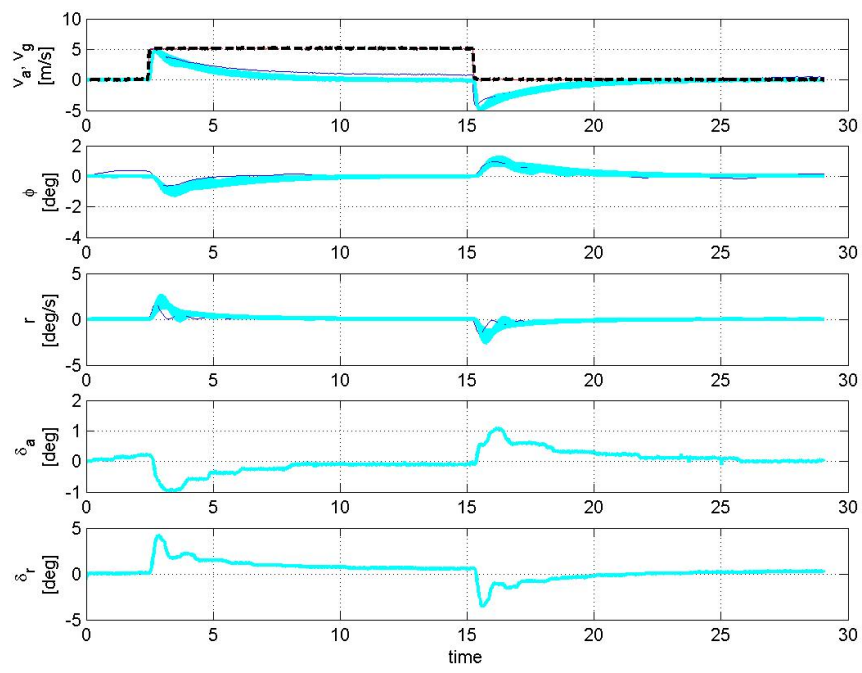

Fig. 8: TRSP UoB-K: CLP gust suppression for $E A S=[100$ : $10: 140]$ kts with random delay $\Delta \in[ \pm 1]$ (15 runs per EAS)

In addition to the above tests, individual and simultaneous doublets for the $z_{m}$ input (the output of the mimicked aircraft, i.e. block $M$ in Fig. 2) were used for a range of magnitudes. Fig. 9 shows the TRSP for strong (10 degrees) doublets in both input channels. The response of UoB-K is given on the left and that of JAXA-K on the right. Notice that the effect on the sway speed (top plots) is similar, but those for the roll angle (middle plots) and yaw rate (bottom plots) is much different -notice the scales. First of all, a nonminimum phase behavior is observed in both (as expected since there is a zero at the origin arising from the roll rate transfer functions), but while for UoB-K the excursions are at worst about $30 \%$, for the baseline controller they are sometimes above $150 \%$. Similarly, it is clear that the yaw rate response of the baseline controller suffers from a more unwanted oscillation across EAS. Finally, although almost unnoticeable, there is a slightly more sluggish and less tighter roll angle tracking behavior for UoB-K. This was considered negligible but as it will be seen later on it was an early indication of a slightly lower turn-coordination capability (which was not a stated design requirement).

\section{B. Design Verification: Hardware-In-The-Loop}

The hardware-in-the-loop (HIL) verification activities for MuPAL- $\alpha$ are actually performed with the full aircraft in the hangar, connecting the FBW computer directly to a control post from where wind and gust excitation signals can be introduced. Of course, before the HILS tests can be 

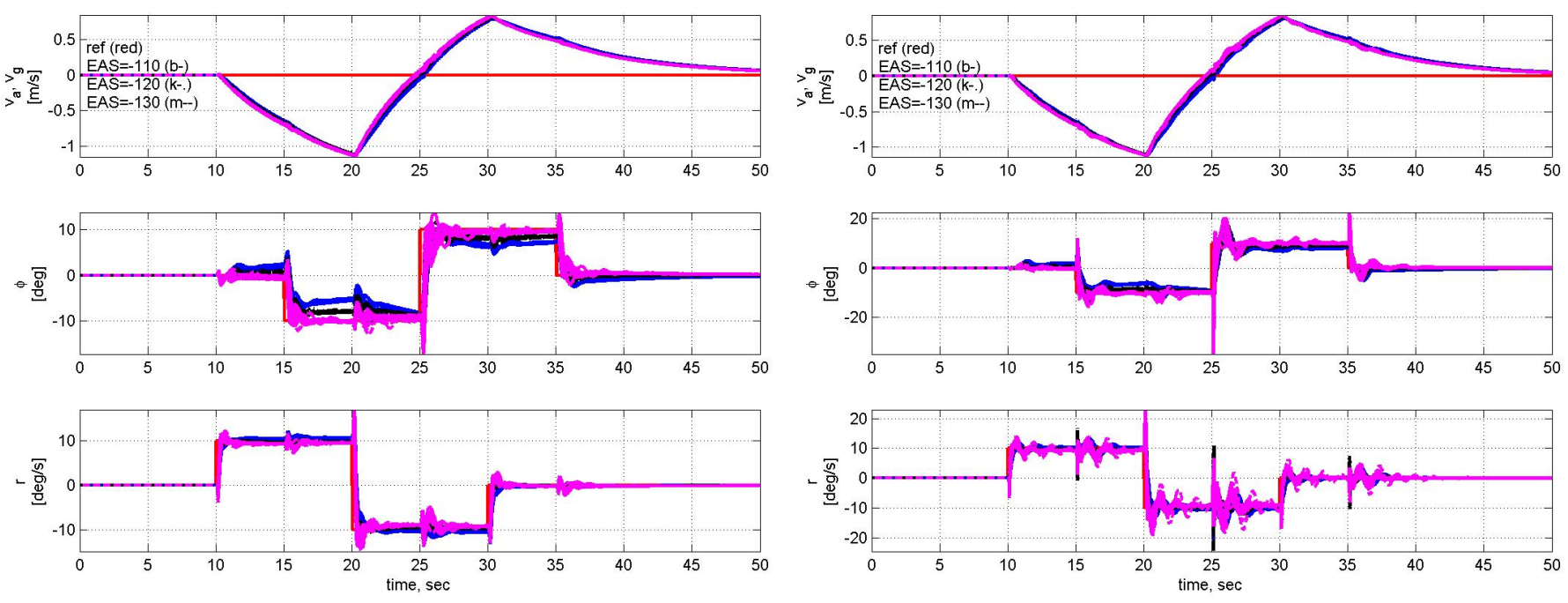

Fig. 9: TRSP: doublets, EAS=[110,120,130] kts with 15 random delays $\Delta \in[ \pm 1]$ per EAS, UoB-K (left) and JAXA-K (right)

carried out the designed flight controllers must be ported to the correct flight $\mathrm{C}$-code format. This porting includes implementing the designs as discrete-time systems (using a bilinear transform with a time constant of 0.02 seconds). In addition, noise reduction filters $F_{1}=\frac{1}{(0.03 s+1)^{3}} \mathbf{I}$ for $u_{m}$ and $F_{2}=\frac{1}{0.02 s+1} \mathbf{I}$ for $y_{p}$ are used (both also as discrete-time systems at the same sampling time but using a zero-order hold).

Then, and similarly to [14], HIL simulations were carried out first by the authors as part of a pre-verification activity, and then after analysis of the resulted data, the proper HIL verification activity was carried out by JAXA test pilots. These HIL simulations [9] confirmed that the designed controllers achieved gust suppression as well as a wide range of maneuverability (specifically, moderate $\phi=20^{\circ}$ and aggressive $\phi=30^{\circ}$ coordinated turns and S-turns) without requiring redesign. The observation from the evaluation pilot (EP) was that both controllers were mostly indistinguishable, although UoB showed a tendency to require "stepping-onthe-ball", which is flight jargon to refer to a decrease of turn-coordination capability (recall the last comment from the previous sub-section).

Fig. 10 shows one set of results where gust was introduced and a moderate maneuver consisting of a coordinated "eight" was performed (i.e. a 360 degrees turn in one direction continued by another in the other direction). The EAS speed for the tests were the same as that for the controller designs (although of course the HIL set-up does not account for the associated aerodynamic force effects). In the figure, as well as in all the rest of the figures, there are two responses: the dashed black showing the response of MuPAL- $\alpha$ and the solid red showing that for the system $M$ of Fig. 2 -i.e. the aircraft being mimicked, which in this case is a B747 aircraft.

\section{Design Validation: Flight tests}

Flight experiments were carried out during two days of flight tests on the $20^{t h}$ and $21^{s t}$ of December 2016 near
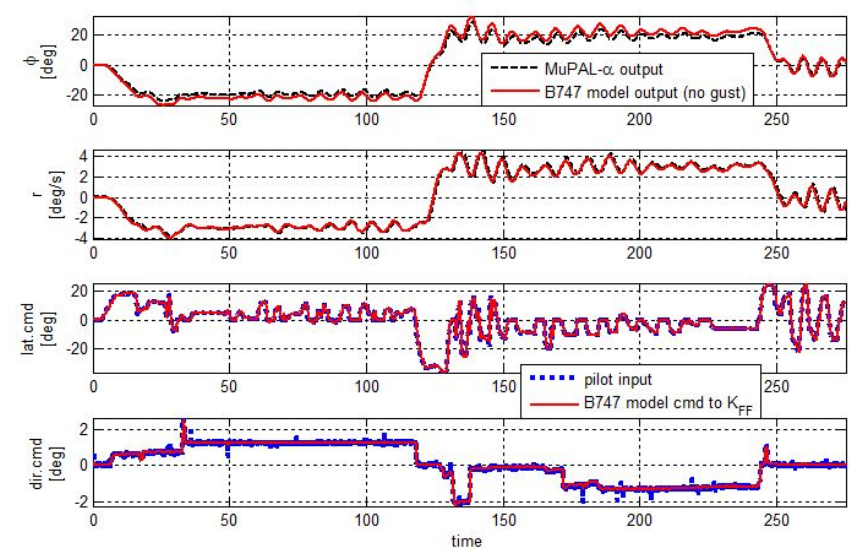

Fig. 10: HILS UoB-K: moderate $360^{\circ}$ steady-turns at $136 \mathrm{kts}$

Tokyo, Japan. As for the HIL, the flight tests followed a manoeuvres' sequence involving moderate and aggressive "eights" and S-turns (sets of 3 turns), starting always with the moderate. The controllers were alternated during the tests to avoid that the EP pilot adapted his response, i.e. first the UoB-K "eight" followed by JAXA-K's "eight" and then similarly with the S-turns. The first day flew the tests at the same EAS used for the designs (136 kts), while the second tested the controllers at $E A S=[110,150] k t s$.

Fig. 11 shows the same maneouver as Fig. 10 but at $E A S=110 \mathrm{kts}$. Notice that both controllers show quite similar behaviour, with some more oscillation on $\phi$ tracking for UoB-K as well as a bit more use of the directional rudder command (i.e. the pilot's pedal). The rest of the tests showed similar responses.

The evaluation pilot's comments were:

- The behaviour is almost indistinguishable.

- At certain speeds/manoeuvres UoB-K feels better.

- At others, UoB-K requires a bit "stepping-on-the-ball".

This is in agreement with the observations during the HIL verification tests, but as aforementioned this effect was not 

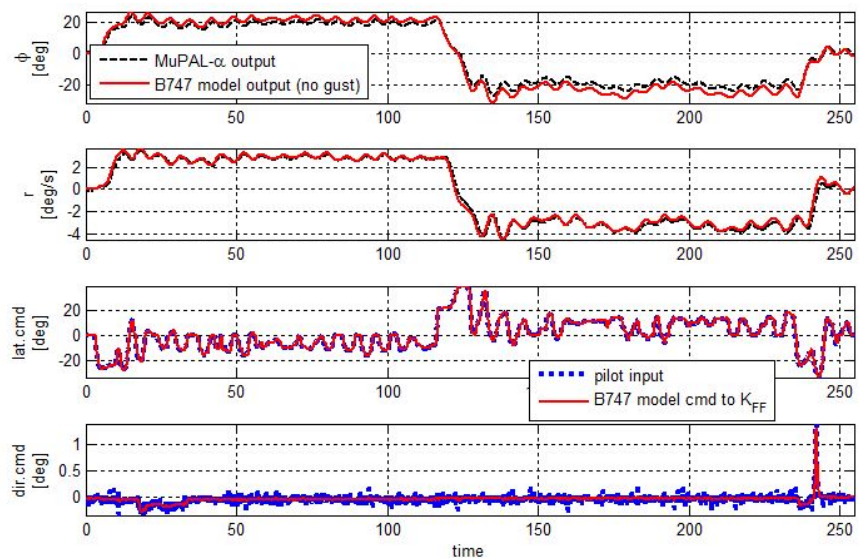

Fig. 11: Flight: moderate $360^{\circ}$ steady-turns at $110 \mathrm{kts}$, UoB-K (left) and JAXA-K (right) observed during the design analysis phase (see Fig. 9). This is the result of a decrease in turn-coordination capability for UoB-K as this was not designed for or indirectly taken into account during the design phase. It is noted that no specific design requirements were set due to JAXA's aim of providing as much design freedom to the VISION consortium, and although the same objectives and interconnection was used JAXA's team has a 10 years flight testing experience with MuPAL- $\alpha$ (as opposed to this being the first piloted flight test experience for UoB team).

\section{CONCLUSIONS}

In this article the results of the (to the best of the authors, first time) piloted flight testing of a structured $H_{\infty}$ controller have been presented. The results show that the controller performed almost equal to a baseline controller. More importantly, it is shown that structured $H_{\infty}$ can use the legacy knowledge (an important concern in the aeronautical industry) while yielding much less complexity, i.e. half the dimension of the baseline controller used for design.

\section{ACKNOWLEDGMENT}

The authors thanks the support of JAXA's flight testing team, especially Mr. Hosoya Tomoaki for his continuous support with the on-ground HIL and flight activities, Mr. Yasuo Uetake and Mr. Shin-ichi Morokuma for their impeccable work as evaluation and safety pilots respectively, and $\mathrm{Mr}$. Naruoka and Mr. Kawaguchi for supporting this work.

\section{REFERENCES}

[1] K. Masui, and Y. Tsukano, Development of a New in-Flight Simulator MuPAL- $\alpha$, AIAA GNC conference, Paper 2000-4574, Aug. 2000.

[2] M. Sato and T. Hagiwara: ILS Approach Flight Experiment of MuPAL- $\alpha$ Using Feedforward Model-Matching Control for Handling Characteristics Realization, Trans. of the Japan Society for Aeronautical and Space Sciences, Vol. 59, No. 11, pp. 315-318, 2011 (in Japanese).

[3] Y. Abe, et al.: Parameter Estimation of Aircraft Dynamics Based on Gray-box Modeling, Proc. of the 54th Aircraft Symposium, JSASS2016-5088, 2016 (in Japanese).

[4] Y. Okuno, et al.: Research on IFR operations for helicopters, Aeronautical and Space Sciences Japan, Vol. 56, No. 9, pp. 240-246, 2008 (in Japanese).
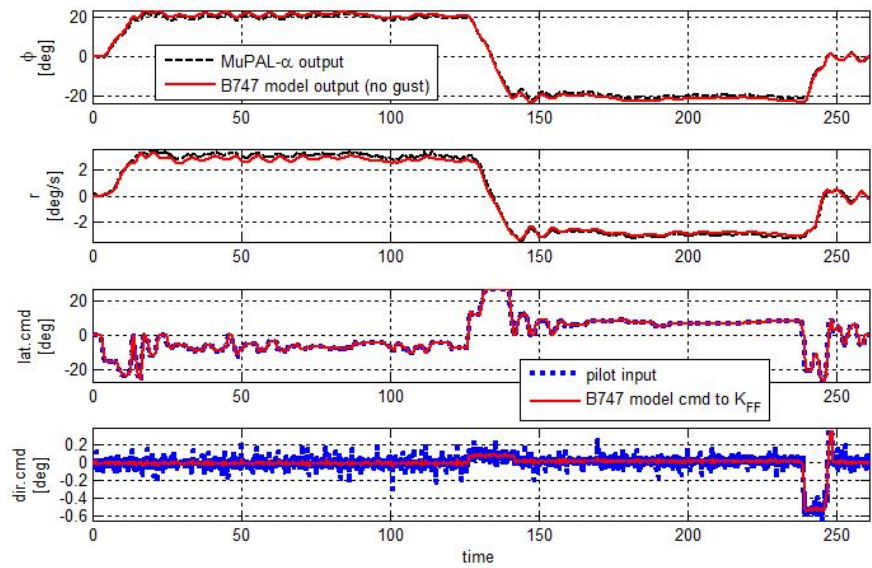

[5] N. C. Weingarten, "History of In-Flight Simulation at General Dynamics," Journal of Aircraft, Vol. 42, No. 2, 2005, pp. 290-298.

[6] M. Sato and A. Satoh, "Simultaneous Realization of Handling and Gust Responses: In-Flight Simulator Controller Design," Journal of Guidance, Control, and Dynamics, Vol. 31, No. 6, 2008, pp. 15451560.

[7] P. Goupil and A. Marcos, "The European ADDSAFE project: Industrial and academic efforts towards advanced fault diagnosis", Control Engineering Practice, Vol. 31, pp. 109-125, 2014.

[8] P. Goupil, J. Boada-Bauxell, A. Marcos, E. Cortet, M. Kerr, and H. Costa, "Airbus efforts towards advanced real-time Fault Diagnosis and Fault Tolerant Control", 19th IFAC World Congress, Cape Town, South Africa, August 2014

[9] A. Marcos and M. Sato, Robust Model-Matching Controller Design Using Matlab "hinfstruct" Command The 4th Multi-symposium on Control Systems (MSCS17), Okayama, Japan

[10] P. Apkarian, D. Noll, and A. Rondepierre: Mixed $H_{2} / H_{\infty}$ Control via Nonsmooth Optimization, SIAM J. on Control and Optimization, Vol. 47, No. 3, pp. 1516-1546, 2008.

[11] G. Puyou and P. Ezerzere: Tolerance of aircraft longitudinal control to the loss of scheduling information -towards a performance oriented approach, IFAC Symposium on Robust Control Design, Denmark, 2012.

[12] H. Lhachemi, D. Saussie and G. Zhu: A Robust and Self-Scheduled Longitudinal Flight Control System: A Multi-Model and Structured $H_{\infty}$ Approach, AIAA SciTech 2014 Conference, Minneapolis, 2012.

[13] P. Apkarian and D. Noll: Nonsmooth $H_{\infty}$ Synthesis, IEEE. Trans. Automatic Control, Vol. 51, No. 1, pp. 71-86, 2006.

[14] M. Sato and A. Satoh: Flight Control Experiment of MultipurposeAviation-Laboratory- $\alpha$ In-Flight Simulator, J. Guidance, Control, and Dynamics, Vol. 34, No. 4, pp. 1081-1096, 2011.

[15] A. Falcoz, C. Pittet, S. Bennani, A. Guignard, C. Bayart, and B. Frapard, Systematic design methods of robust and structured controllers for satellites, Space Journal, vol. 7, no. 3, pp. 319 334, 2015.

[16] C. Pittet and P. Prieur, Structured Accelero-Stellar Estimator for Microscope Drag-Free Mission, Advances in Aerospace Guidance, Navigation and Control: Selected Papers of the Third CEAS Specialist Conference on Guidance, Navigation and Control held in Toulouse, pp. 591-604, 2015

[17] L. Coutard, F. Chaumette and J.M. Pflimlin, Automatic landing on aircraft carrier by visual servoing, IEEE/RSJ International Conference on Intelligent Robots and Systems, 2011

[18] S. Suzuki, F. Kawamura and K. Masui, Autonomous Flight Control and Guidance System of Accident Aircraft, 24th International Congress of the Aeronautical Sciences, 2004

[19] S. Suzuki et al., Flight Demonstration of Fault Tolerant Flight Control System, 28th International Congress of the Aeronautical Sciences, 201

[20] P. Goupil and A. Marcos, Industrial perspectives for aerospace applications, chapter 19 in GARTEUR FM-AG16, Lecture Notes in Control and Information Science, Springer-Verlag, 2010. 